\title{
Initial Single-Site Surgical Experience with SMILE: A Comparison of Results to FDA SMILE, and the Earliest and Latest Generation of LASIK
}

\author{
Majid Moshirfar · Michael S. Murri • Tirth J. Shah • Steven H. Linn • \\ Yasmyne Ronquillo • Orry C. Birdsong · Phillips C. Hoopes Jr.
}

Received: May 13, 2018 / Published online: June 29, 2018

(C) The Author(s) 2018

\section{ABSTRACT}

Introduction: The primary objective was to show our initial surgical single-site experience with small incision lenticule extraction (SMILE) after the official enrollment in March 2017 following Food and Drug Administration (FDA) approval for simple myopia in late 2016 in the United States and, subsequently, compare our results to the earliest and most advanced generation of excimer platforms for laser-assisted in situ keratomileusis (LASIK) surgery.

Methods: This was a retrospective single-site study of 68 eyes from 35 patients who had SMILE surgery. The patients' preoperative and

Enhanced digital features To view enhanced digital features for this article go to https://doi.org/10.6084/ m9.figshare.6572846.

M. Moshirfar $(\bowtie) \cdot$ M. S. Murri

Department of Ophthalmology and Visual Sciences, John A. Moran Eye Center, University of Utah

School of Medicine, Salt Lake City, UT, USA

e-mail: cornea2020@me.com

M. Moshirfar

Utah Lions Eye Bank, Murray, UT, USA

M. Moshirfar · S. H. Linn · Y. Ronquillo .

O. C. Birdsong · P. C. Hoopes Jr.

HDR Research Center, Hoopes Vision, Draper, UT, USA

T. J. Shah

University of Arizona College of Medicine, Phoenix, Phoenix, AZ, USA postoperative uncorrected distance visual acuity (UDVA), corrected distance visual acuity (CDVA), manifest sphere, manifest cylinder, intraoperative complications, and preoperative and postoperative visual symptoms were collected. We compared our findings to the results from the FDA SMILE study, and to the three earliest (1999-2000) and three of the most updated (2013-2016) platforms for LASIK.

Results: The cumulative UDVA was $20 / 20$ and $20 / 40$ or better in $74 \%$ and $100 \%$ of patients, respectively. The intended target refraction was within \pm 0.5 and $\pm 1.00 \mathrm{D}$ in $80 \%$ and $93 \%$ of cases, respectively. The prevalence of dry eyes decreased by nearly half from 1-week to the 6-month postoperative interval. Patients noted improvement in glare (17\%), halos (17\%), fluctuation (25\%), and depth perception (8\%) at the 6-month interval compared to preoperative levels. Conclusions: This study's findings are consistent with current SMILE reports. Notably, the results are superior to the earliest generation of LASIK, however inferior to the latest excimer platforms. SMILE does meet the efficacy and safety criteria met by FDA; however, there is a definite need for further improvement to reach the superior refractive outcomes produced by the latest generation of LASIK platforms.

Keywords: Alcon Contoura; SMILE astigmatism; SMILE dry eyes; SMILE improvements; SMILE vs. LASIK; SMILE 
symptoms; Visumax femtosecond laser; VISX iDesign; Wavefront

\section{INTRODUCTION}

Lenticular extraction of the corneal stroma was first conceived in the late 1990s with the creation of the lenticule with a picosecond laser $[1,2]$ followed by its manual extraction. Later, Femtosecond Lenticule Extraction (FLEx) was innovated, with the purpose of treating patients with severe myopia. With the advent of the Visumax femtosecond laser (Carl Zeiss Meditec, Jena, Germany), and the refinement of the technique to involve only a $2-3 \mathrm{~mm}$ incision, small incision lenticule extraction (SMILE) became an increasingly viable procedure in mainstream refractive surgery $[3,4]$. FDA registered clinical trials began in 2011 and official trials for SMILE approval began in the United States in 2012.

Since that time, numerous international studies originating in France, Singapore, Egypt, China, Spain, Japan, Germany, Denmark, South Korea, Turkey, India, and the United Kingdom have reported on SMILE visual outcomes [4-22]. However, with FDA approval in September of 2016, and official enrollment of patients in the United States in early 2017, there is a paucity of data from patients in the United States. Our objective was to report the 6-month efficacy, safety, predictability, and stability of patients undergoing SMILE surgery from a single surgical center. Because there is limited literature regarding the visual quality after SMILE, our second aim was to collect data concerning patient symptoms (i.e., glare, dryness, halos, etc.) after surgery. Lastly, given that SMILE is still a relatively new surgery in the United States, we compared our findings to those from the earliest excimer platforms for LASIK (1999-2000) and to the most updated excimer platforms for LASIK (2013-2016).

\section{METHODS}

This retrospective study was conducted at the HDR research center, Hoopes Vision, Draper,
UT, USA. The study included 68 eyes from 35 patients. All procedures performed in studies involving human participants were in accordance with the ethical standards of the institutional and/or national research committee and with the 1964 Helsinki declaration and its later amendments or comparable ethical standards. Informed consent was obtained from all individual participants included in the study. This study was approved by the Hoopes, Durrie, Rivera Research Review Board. Before providing written informed consent, all patients were given detailed information regarding each treatment. The preoperative manifest sphere and cylinder of our preoperative patient population ranged from $-7.50 \mathrm{D}$ to $-2.75 \mathrm{D}$ and $-0.75 \mathrm{D}$ to $0.00 \mathrm{D}$, respectively. Patients had complete refractive screening and normal fundoscopic exam prior to enrollment in the study. All candidates were healthy and appropriate refractive surgical candidates for SMILE. Any patient with cylinder greater than $0.75 \mathrm{D}$, abnormal corneal topographic findings (i.e., keratoconus, pellucid marginal degeneration, etc.), history of herpes zoster ophthalmicus or herpes simplex keratitis, significant dry eye syndrome refractory to treatment or dry eye disease, history of diabetes or connective tissue disease, pregnant or lactating were excluded from our study.

SMILE was performed using the Visumax $500 \mathrm{kHz}$ femtosecond laser (Carl Zeiss Meditec, Jena, Germany) by two surgeons (MM, PCH). Although the surgeons had no prior SMILE experience, they have each had LASIK experience of over 15 years. Follow up appointments occurred at 1 day, 1 week, 1 month, 3 months, and 6 months. At the 1-week interval, we lost one patient (two eyes) to follow-up. We had 66 eyes and 41 eyes of follow-up data at the 3-month and 6-month interval, respectively. Some patients have not had their 6-month follow-up, thus explaining the difference between the 3-month and 6-month sample size. A few of our patients had their 1-year follow-up; however, because this was a small subset of our cohort ( $\sim 10$ eyes), we decided to omit these data from our study. Data collected included patients' preoperative and postoperative uncorrected distance visual acuity (UDVA), 
corrected distance visual acuity (CDVA), manifest sphere, manifest cylinder, and any complications during or after surgery. LogMAR visual acuity was calculated with count fingers (CF) set at $\log$ MAR 2.0 per recommendation by Holladay [23]. Three eyes were excluded from UDVA analysis because their target refraction was adjusted for monovision, but were included in all other visual and refractive analyses. Surgical parameters were set with a cap thickness of $120 \mu \mathrm{m}$ and cap diameter of $7.5 \mathrm{~mm}$. Hinge placement was superior with hinge angle at 50 degrees and side-cut angle of 90 degrees. Lenticule diameter was $6.5 \mathrm{~mm}$. Spot separation was $3 \mu \mathrm{m}$ for the lenticule, $2.5 \mu \mathrm{m}$ for the lenticule side-cut, $3 \mu \mathrm{m}$ for the flap, and $2 \mu \mathrm{m}$ for the flap side cut. Laser-bed energy was set at 145 nJ.

After obtaining our standard refractive results, the percent of individuals within 20/20 UDVA, 20/40 UDVA, $\pm 0.5 \mathrm{D}$ MRSE, and $\pm 1.0 \mathrm{D}$ MRSE were compared to those published by the recent FDA SMILE study [24]. We also compared our findings to the first (1999-2000) and latest generation (2013-2016) of excimer LASIK platforms. The following early generation LASIK platforms were used for comparisons: SVS Apex Plus Excimer Laser Worksation by Summit Technology, Inc. (Waltham, MA, USA; PMA: P930034/S13) [25], Nidek EC5000 Excimer Laser System by Nidek Technologies, Inc. (Pasadena, CA, USA; PMA: P970053/S002) [26], and VISX STAR S2 Excimer Laser System by AMO Manufacturing (Scottsdale, AZ, USA; PMA: P990010) [27]. We chose these specific earlier excimer models because they represented the most popular platforms at that time point in the United States. The following newest LASIK platforms were used for comparisons: STAR S4 Excimer Laser System with iDesign Advanced WaveScan Studio System by VISX, Inc. (Milpitas, CA, USA; PMA: P930016/S045) [28], Nidek EC-5000 Excimer Laser System by Nidek, Inc. (Gamagori, Aichi, Japan; PMA: P970053/S011) [29], and Allegretto Wave Eye-Q Excimer Laser by Alcon Laboratories, Inc. (Fort Worth, TX, USA; PMA: 020050/S12) [30].

At the preoperative appointment and each follow-up visit ( 1 week, 1 month, 3 months, and
6 months), we assessed for the presence or absence of any dry eye symptoms via a physician-interview for a total of 22 patients. For example, prior to the surgery, each patient was asked whether or not they have any dry eye symptoms with a "yes or no" response. At the 6-month postoperative interval, the same question was asked to the patient with a "yes or no" response. The specific severity of the dry eyes was not obtained. For further analysis, we then performed corneal staining to assess for superficial punctate keratitis (SPK) for the same 22 patients. We assessed SPK via a scoring scale: 0 indicating no staining, $1+$ indicating mild staining, 2 + indicating moderate staining, and $3+$ indicating significant staining of cornea.

At the 6-month postoperative visit, a total of 24 patients were asked via a physician-interview whether their visual symptoms concerning glares, halos, starbursts, double vision, fluctuation, and depth perception were "better, worse, or not changed" from the preoperative levels.

We assessed differences in visual outcomes between the first 12 patients and last 12 patients to receive SMILE surgery. The postoperative visual outcomes that were assessed include: manifest sphere, cylinder, and spherical equivalence. Lastly, in order to appropriately pool data from both surgeons, we ensured there was no statistically significant difference in the aforementioned postoperative visual outcomes between surgeons.

\section{Statistical Analysis}

Data was collected and analyzed using Datagraph-Med (Wendelstein, Germany) and Microsoft Excel software. Astigmatic data was calculated using Dr. Peyman's online Astigmatic Vector Analyzer [31]. Standard refractive graphs were created using Standard Graphs for Reporting Refractive Surgery (London Clinic) [32]. The postoperative sphere, cylinder, and spherical equivalence of the first 12 patients was compared to the last 12 patients using a twopair Wilcoxon signed-rank test. The same test was used to compare results between different surgeons. A $p$ value less than 0.05 was considered statistically significant. 


\section{RESULTS}

The average patient age was 31 years with a standard deviation of 7 and a range from 23 to 49 years of age. At 6 months, we had 21 right eyes and 20 left eyes from 63\% female and 37\% male patients. Average logMAR UDVA 6 months after surgery was $0.05 \pm 0.13$ and spherical equivalent (SE) 6 months after surgery was $-0.25 \pm 0.53 \mathrm{D}$ (Table 1). The 6-month UDVA, manifest sphere, manifest SE, and CDVA were all significantly improved $(p<0.0001$; Table 1). The manifest cylinder significantly increased from $-0.23 \mathrm{D}$ at preoperative to $-0.49 \mathrm{D}$ at 6 months postoperative $(p<0.0001$; Table 1$)$. The standard refractive surgery graphs are shown in Fig. 1.

With regards to efficacy, cumulative UDVA was $20 / 20,20 / 25$, or $20 / 40$ or better in $74 \%$, $87 \%$, and $100 \%$ of patients, respectively. The UDVA at 6 months was the same or better than pre-operative CDVA in $74 \%$ of cases and within one line of CDVA in $87 \%$ of cases. The efficacy index was 0.89 . At 6 months, $37 \%$ of patients gained one Snellen line of CDVA.

Concerning safety, when comparing pre-operative CDVA to postoperative CDVA, $63 \%$ of patients had no change and no eye had a decrease in CDVA. The safety index was 1.12.

With regards to predictability, the intended target refraction was within \pm 0.5 and $\pm 1.00 \mathrm{D}$ in $80 \%$ and $93 \%$ of cases, respectively. We noticed there was an induction of astigmatism. For instance, refractive astigmatism increased in the $0.51-0.75 \mathrm{D}$ surgical subgroup ( $2 \%$ preoperative to $17 \%$ postoperative) and $0.76-1.00 \mathrm{D}$ surgical subgroup (0\% preoperative to $7.3 \%$ postoperative). We also noticed no pattern in the refractive astigmatism angle of error. About half the eyes had a positive angle of error and vice versa.

Lastly, concerning stability, $7 \%$ of patients had greater than $0.5 \mathrm{D}$ change in refraction over the postoperative period from 1 month to 6 months. Overall, the greatest improvement in visual acuity and refraction was seen in the first month postoperatively without significant changes in the 3-month and 6-month followup.

\section{Results vs. FDA SMILE Data}

Visual acuity outcomes in our study were UDVA of $20 / 20$ and $20 / 25$ or better in $74 \%$ and $83 \%$ of patients and $20 / 40$ or better in $98 \%$ of patients.

Table 1 Describes changes in standard visual outcomes from preoperative to the 1-month, 3-month, and 6-month interval

\begin{tabular}{|c|c|c|c|c|}
\hline & Pre-operative $(n=68)$ & $\begin{array}{l}\text { 1-month } \\
\text { Postoperative } \\
(n=66)\end{array}$ & $\begin{array}{l}\text { 3-month } \\
\text { Postoperative } \\
(n=62)\end{array}$ & $\begin{array}{l}\text { 6-month } \\
\text { Postoperative } \\
(n=41)\end{array}$ \\
\hline $\begin{array}{l}\text { UDVA } \\
\qquad(\log M A R)\end{array}$ & $\begin{array}{l}1.46 \pm 0.33 \text { (0.88 to } \\
2.00)\end{array}$ & $\begin{array}{l}0.09 \pm 0.16(-0.10 \text { to } \\
0.80)\end{array}$ & $\begin{array}{l}0.05 \pm 0.14(-0.10 \text { to } \\
0.54)\end{array}$ & $\begin{array}{l}0.05 \pm 0.13(-0.12 \text { to } \\
0.48)^{\mathrm{a}}\end{array}$ \\
\hline $\begin{array}{l}\text { CDVA } \\
\qquad(\log M A R)\end{array}$ & 0 & $\begin{array}{l}-0.01 \pm 0.06(-0.10 \\
\quad \text { to } 0.18)\end{array}$ & $\begin{array}{l}-0.02 \pm 0.05(-0.10 \\
\quad \text { to } 0.18)\end{array}$ & $\begin{array}{l}-0.05 \pm 0.06(-0.12 \\
\quad \text { to } 0.00)^{\mathrm{a}}\end{array}$ \\
\hline Manifest SE (D) & $\begin{array}{l}-5.16 \pm 1.34(-7.50 \\
\quad \text { to }-2.75)\end{array}$ & $\begin{array}{l}-0.19 \pm 0.53(-1.50 \\
\quad \text { to } 1.38)\end{array}$ & $\begin{array}{l}-0.21 \pm 0.46(-1.75 \\
\quad \text { to } 1.13)\end{array}$ & $\begin{array}{l}-0.26 \pm 0.53(-1.63 \\
\quad \text { to } 1.25)^{\mathrm{a}}\end{array}$ \\
\hline $\begin{array}{l}\text { Manifest sphere } \\
\text { (D) }\end{array}$ & $\begin{array}{l}-5.05 \pm 1.32(-7.50 \\
\quad \text { to }-2.75)\end{array}$ & $\begin{array}{l}0.01 \pm 0.54(-1.25 \text { to } \\
1.75)\end{array}$ & $\begin{array}{l}0.00 \pm 0.48(-1.50 \text { to } \\
1.75)\end{array}$ & $\begin{array}{l}-0.01 \pm 0.58(-1.50 \\
\quad \text { to } 1.75)^{\mathrm{a}}\end{array}$ \\
\hline $\begin{array}{l}\text { Manifest } \\
\text { cylinder (D) }\end{array}$ & $\begin{array}{l}-0.23 \pm 0.22(-0.75 \\
\quad \text { to } 0.00)\end{array}$ & $\begin{array}{l}-0.41 \pm 0.44(-2.50 \\
\quad \text { to } 0.25)\end{array}$ & $\begin{array}{l}-0.42 \pm 0.36(-1.50 \\
\quad \text { to } 0.00)\end{array}$ & $\begin{array}{l}-0.49 \pm 0.30(-1.50 \\
\quad \text { to } 0.00)^{\mathrm{a}}\end{array}$ \\
\hline
\end{tabular}

The data represent the mean \pm standard deviation (range)

${ }^{a}$ Indicates statistically significant findings $(p<0.05)$ 


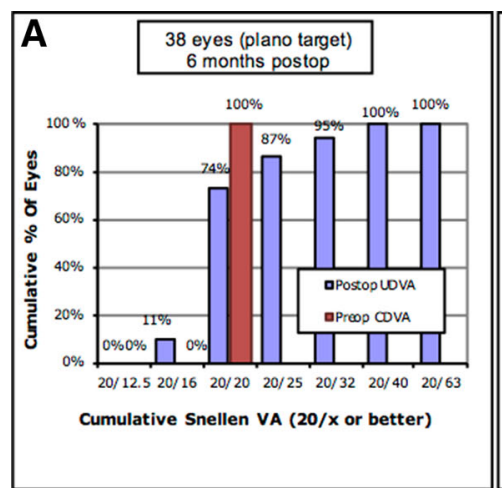

Uncorrected Distance Visual Acuity

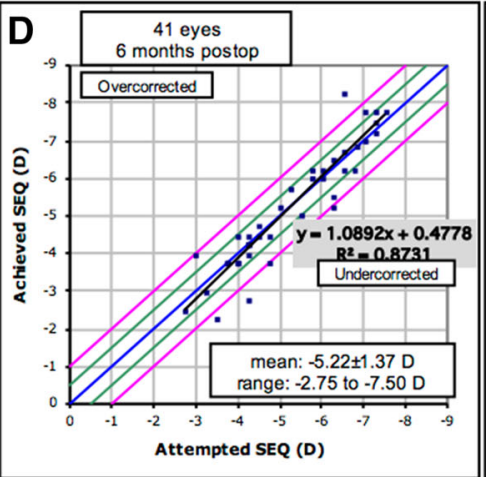

Spherical Equivalent Refraction Attempted vs. Achieved

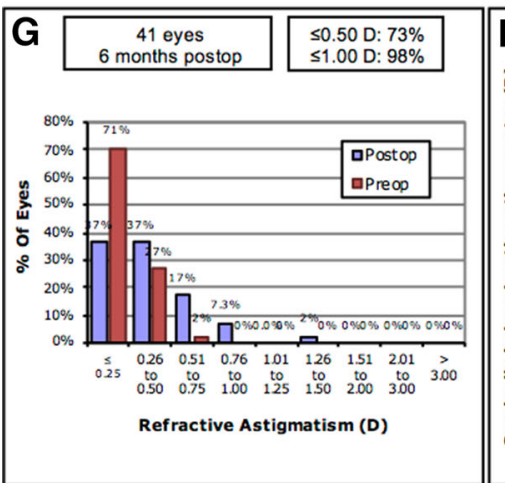

Refractive Astigmatism

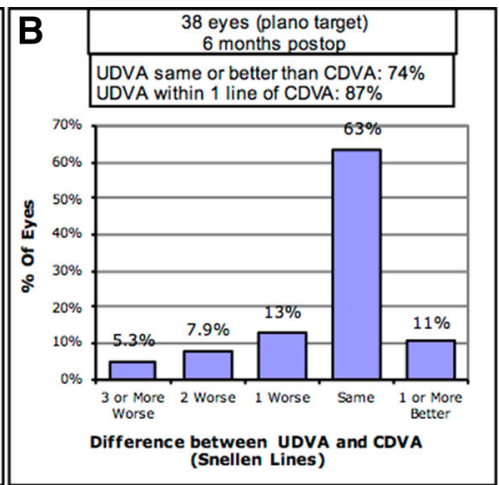

Uncorrected Distance Visual Acuity vs. Corrected Distance Visual Acuity

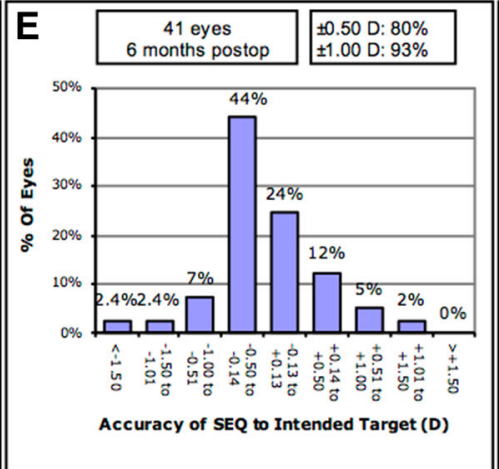

Spherical Equivalent Refraction Accuracy

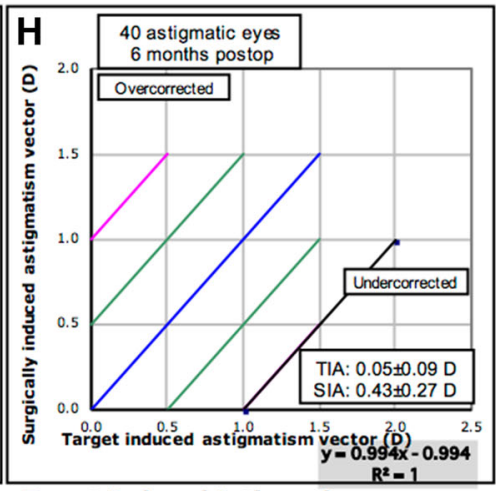

Target Induced Astigmatism vs. Surgically Induced Astigmatism
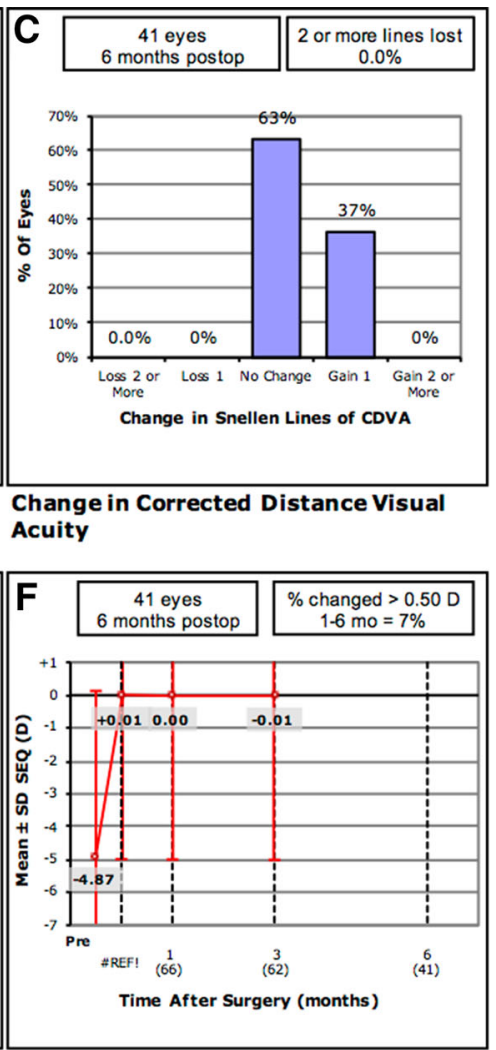

Sphereical Equivalent Refraction Stability

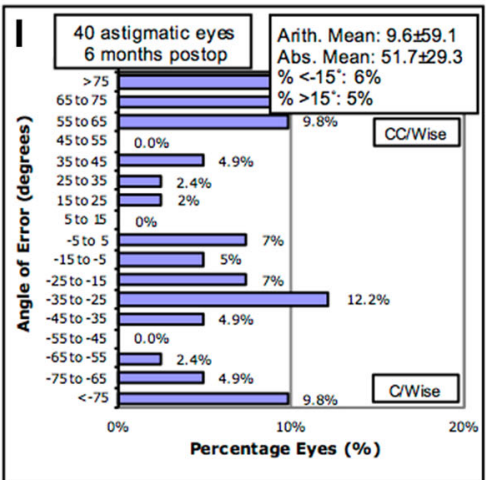

Refractive Astigmatism Angle of Error

Fig. 1 Standard refractive surgery graphs

These can be compared to recent FDA pre-market approval (PMA) data for SMILE in the United States [24] in which $87.5 \%$ and $99.7 \%$ of patients achieved UDVA of $20 / 20$ and $20 / 40$ or better, respectively (Table 2 ). In our study population, patients were within \pm 0.5 and $\pm 1.00 \mathrm{D}$ of target refraction in $80 \%$ and 93\% of cases, respectively. FDA approval data reported values of $93 \%$ within $\pm 0.5 \mathrm{D}$ and $98.5 \%$ within $\pm 1.00 \mathrm{D}$. 
Table 2 A comparison of our SMILE results to those found by the FDA, in addition to the early generation (1999-2000) and most updated excimer platforms (2013-2016)

\begin{tabular}{lllll}
\hline & \% within 20/20 UDVA & \% within 20/40 UDVA & \% within $\mathbf{\pm}$ 0.5 D & \% within + 1.0 D \\
\hline Our SMILE results & 74 & 98 & 80 & 93 \\
FDA SMILE results & 87.5 & 99.7 & 93 & 98.5 \\
\multicolumn{2}{l}{ Current Excimer Laser Platform } & & & \\
VISX iDesign (2016) & 91.7 & 100 & 69 & 93 \\
Alcon Contoura (2013) & 88.9 & 98.7 & 95 & 100 \\
Nidek EC-5000 (2013) & 88.7 & 100 & 91 & 99 \\
Early Excimer Laser Platform & & & 84 \\
SVS Apex Plus (1998) & 46.9 & 92.1 & 61 & 90 \\
Nidek EC-5000 (2000) & 47.4 & 84.4 & 67 & 90.6 \\
VISX STAR S2 (1999) & 54.1 & 95.4 & 72.5 & \\
\hline
\end{tabular}

\section{Results vs. Early Generation LASIK Platforms}

The results showed that overall our SMILE results had significantly more percentage of eyes within 20/20 UDVA, 20/40 UDVA, \pm 0.5 $\mathrm{D}$, and $\pm 1.0 \mathrm{D}$ than those recorded by the early platforms. An average of $51 \%$ and $91 \%$ of patients had UDVA under 20/20 and 20/40, respectively (Fig. 2) compared to $74 \%$ and $98 \%$ of our SMILE patients, respectively.

\section{Results vs. Latest Generation LASIK Platforms}

This study reported 20/20 UDVA or better for $91.7 \%, 88.9 \%$, and $88.7 \%$ of patients and $20 / 40$ vision or better for $100 \%, 98.7 \%$, and $100 \%$ of patients in the Visx iDesign, Alcon Contoura, and Nidek EC-5000, respectively. In addition, achieved refraction was within \pm 0.5 and \pm $1.00 \mathrm{D}$ of target refraction in $69 \%$ and $93 \%$ for Visx, 91\% and 99\% for Nidek CATz, and 95\% and $100 \%$ in the Alcon Contoura, respectively. An average of $89 \%$ and $99 \%$ of patients had UDVA under 20/20 and 20/40, respectively (Fig. 2).

\section{Reported Visual Symptoms}

Our findings showed a decrease in the percentage of dry eyes over time. Our findings showed $100 \%, 88 \%, 45 \%$, and $45 \%$ of reported dry eyes at the 1-week, 1-month, 3-month, and 6-month intervals, respectively ( $n=22$ eyes). Notably, preoperative levels of dry eyes were $45 \%$, thus indicating that at 3 months, the prevalence of dry eyes returned to baseline. To correlate reported dry eyes with exam findings, patients had decreased $1+, 2+$, and 3+ SPK staining over time. The number of eyes without SPK increased from four in the first week to 16 at the 6-month interval ( $n=22$ eyes; Fig. 3). Additionally, we used a physician-centered questionnaire to assess other visual symptoms at the 6-month postoperative interval to assess whether these symptoms have "improved, worsened, or not changed" from preoperative levels (Fig. 4). We found that symptoms of glare (Fig. 4a), halos (Fig. 4b), fluctuation (Fig. 4c), and depth perception (Fig. 4d) were better in 17, 17,25 , and $8 \%$ of eyes at 6 months than preoperative, respectively ( $n=24$ eyes). We found $8 \%$ of eyes had worsening of glare (Fig. $4 \mathrm{a}$ ), halos (Fig. 4b), starburst (Fig. 4c), double vision (Fig. 4d), fluctuation (Fig. 4e), and depth perception (Fig. 4f). Importantly, this $8 \%$ is 


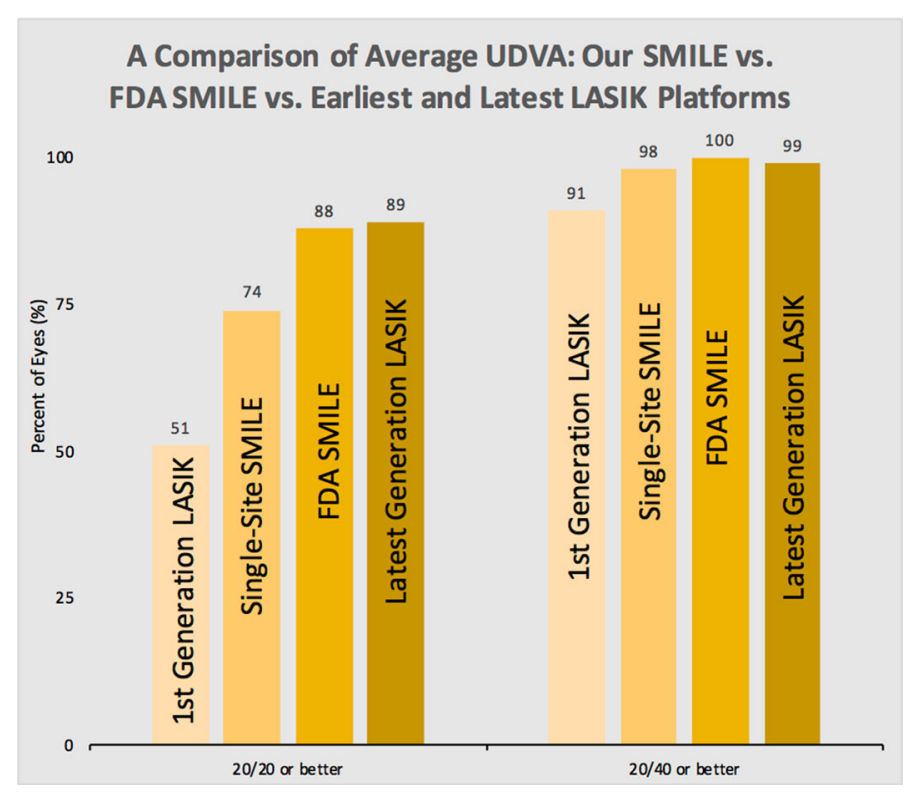

Fig. 2 Comparing the average UDVA between our SMILE results, the FDA SMILE results, and the earliest and latest excimer platforms for LASIK

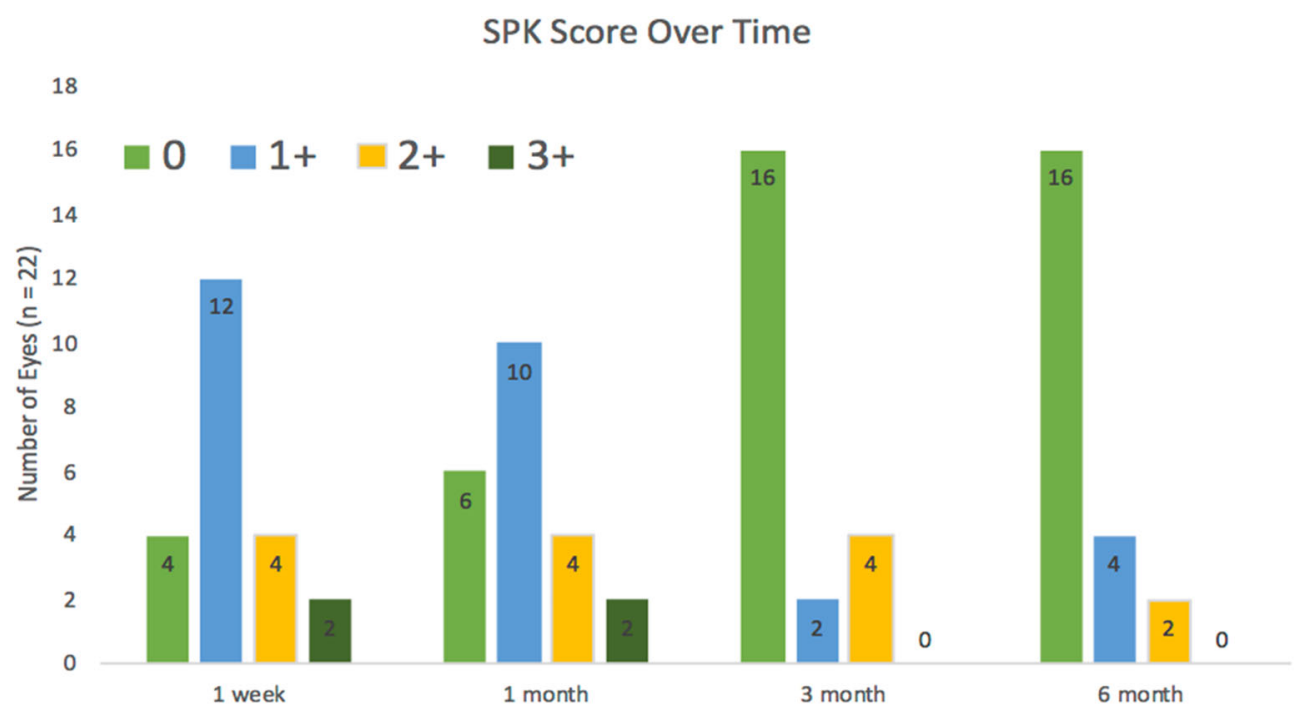

Fig. 3 Changes in superficial punctate keratitis (SPK) scoring from 1-week, 1-month, 3-month, and 6-month postoperative intervals $(n=22)$

composed of one patient (two eyes), who reported worsening of all aforementioned symptoms. It is likely this patient had these symptoms due to moderate SPK and dryness with residual astigmatism.

\section{Visual Outcome Differences between Surgeons}

There was no statistically significant differences in postoperative sphere, cylinder, and spherical equivalence between the two surgeons $(p>0.05)$. 


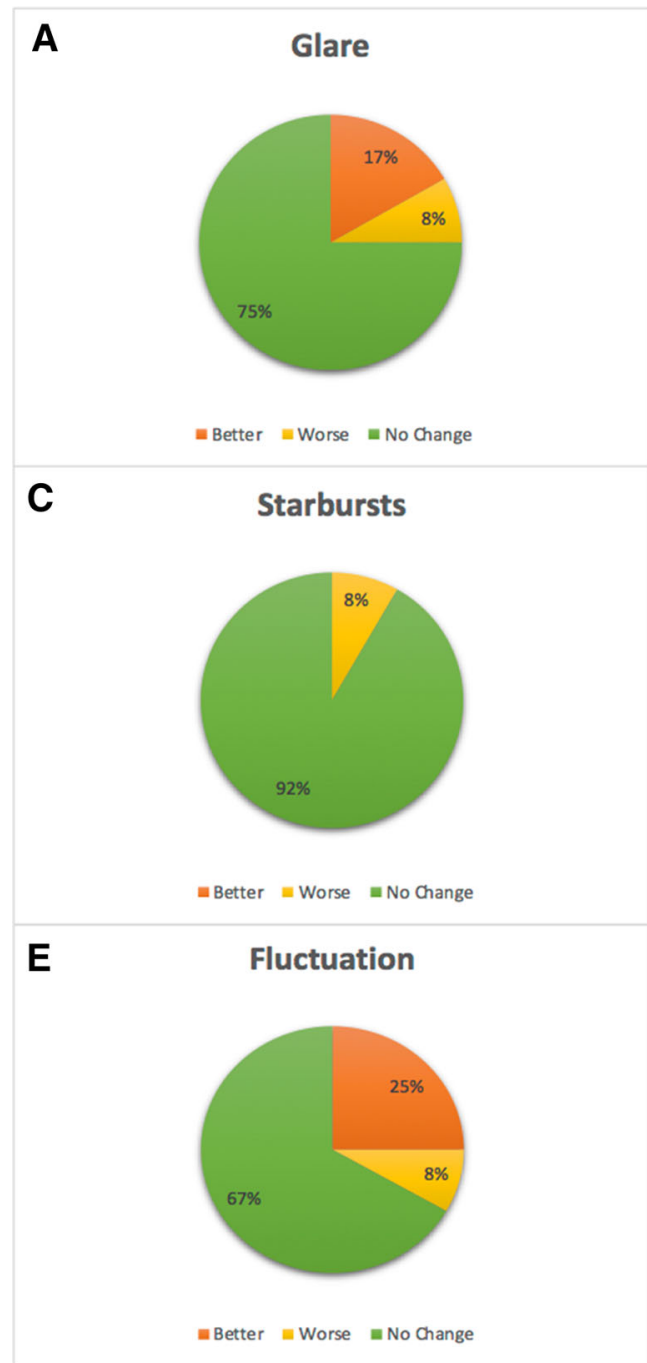

Fig. 4 Patients comparing visual symptoms at 6 months as "better, worse, or no change" from their preoperative levels $(n=24)$. The following symptoms were compared: a glare,

\section{Visual Outcome Differences between First 12 and Last 12 Patients}

The last 12 patients to receive SMILE were found to have spherical equivalence closer to emmetropia $(p=0.0058)$.

\section{Complications}

One patient experienced a difficult lenticule extraction and was subsequently taken from the laser suite to the operating microscope for

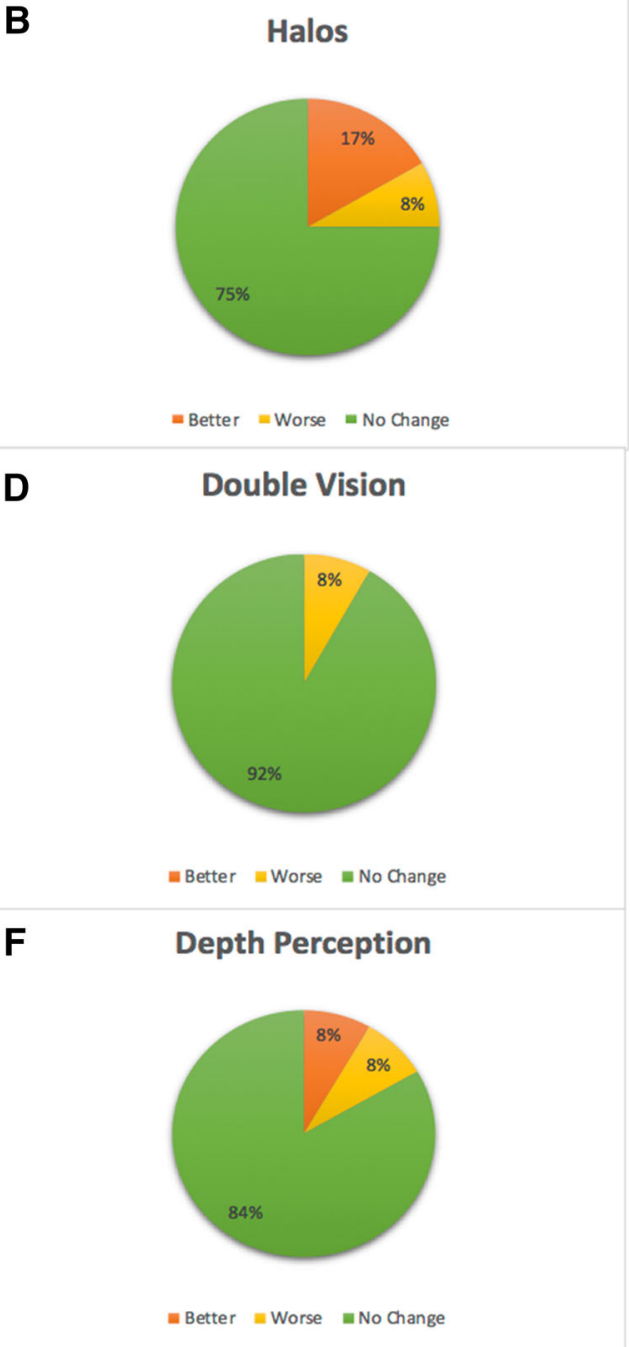

b halos, c starbursts, $\mathbf{d}$ double vision, e fluctuation, $\mathbf{f}$ depth perception

better visualization and uncomplicated removal of the lenticule without further sequelae. There were no other intraoperative complications including cap perforation, incomplete incision creation, or lenticular remnants. Postoperatively, two eyes (5\%) in one patient developed diffuse lamellar keratitis (DLK), which resolved with topical corticosteroid treatment. Three eyes $(7 \%)$ from two patients required postoperative enhancement after the initial 6 months: one patient required unilateral limbal relaxing incisions (LRI) for correction of postoperative cylinder $(-1 \mathrm{D})$ and two eyes from the same 
patient with DLK required bilateral photorefractive keratectomy (PRK) for undercorrection of myopic spherical refraction. There were no occurrences of keratectasia or epithelial downgrowth at the postoperative 6-month visit.

\section{DISCUSSION}

Historically, we remember when earlier excimer generation platforms for LASIK surgery produced subpar results with nearly less than 50\% of our patients achieving 20/20 UDVA at some later postoperative interval. Our SMILE results were superior to these first generation excimer LASIK platforms from 1999 to 2000, yet inferior to the visual outcomes from the newest advanced LASIK platforms from 2013 to 2016. We believe these results must be viewed in the light of years of surgical and computational refinement for LASIK. In fact, a recent review conducted by Sandoval et al. [33] documented the continuous improvement in LASIK outcomes since the first approval of LASIK technology in the late 1990s, corresponding with technological and treatment advances.

As one of the first single-site experiences with SMILE in the United States following the FDA approval, our preliminary results were similar to other SMILE studies. For instance, Sekundo et al. [34] found that $83.5 \%$ and $97.6 \%$ of patients had 20/20 and 20/40 vision or better, respectively. Another study by Shah et al. [5] found that $62 \%$ and $95 \%$ of patients were $20 / 20$ and 20/40 or better, respectively. Other results summarized in a 2014 review by Moshirfar et al. [4] include ranges of $39-96 \%$ within $20 / 20$ and $83-100 \%$ within $20 / 40$ vision. Since that review, a number of new studies have also been published [6-14, 16-22] to which our findings were consistent with UDVA results. Although one study [4] reported as high as $96 \%$ within $20 / 20$ and $100 \%$ within 20/25 UDVA, their patient cohort had an average preoperative SE of $2.61 \mathrm{D}$ compared to the SE $-5.16 \mathrm{D}$ in our study population.

In the United States, SMILE has not been approved for astigmatic correction [24]. Therefore, it is likely that the presence of astigmatism in our study population affected our overall visual outcomes. Notably, our findings showed that SMILE induced astigmatism in a small subset of our cohort with preoperative cylinder greater than $0.26 \mathrm{D}$. Although we don't believe this was the case in our study, a possible and often cited complication of SMILE is an inadvertent lenticule remnant from incidental tearing that can induce irregular astigmatism $[35,36]$. Other than this complication, there was limited prior literature addressing any induction of astigmatism with SMILE; however, past publications have consistently shown SMILE inferior to LASIK with regards to astigmatic corrections [8,37-39]. There are quite a few reasons why SMILE has a tendency to produce these suboptimal outcomes. One is the lack of an automated cyclotorsion alignment that can correct for any unintentional movement of the head and body under the laser [37]. Ganesh et al. [15] showed in his SMILE cohort a cyclotorsion of more than $5^{\circ}$ in approximately $20 \%$ of eyes and demonstrated improved astigmatic outcomes via using preoperative limbal marks as a guide to reduce cyclotorsion [37]. The other reason is a lack of an automated centration control in the Visumax excimer platform [24] by which small misalignments can lead to slight decentration and may impact astigmatism. Until Visumax develops an automated centration and cyclotorsion control software, we believe the technique of manually demarcating the limbus may help improve astigmatic outcomes.

When compared to the recent FDA SMILE results, our visual outcomes were comparable, although not as strong. The patients in our study had negligible preoperative myopic and astigmatic differences from the patient population in the FDA study $(-5.05$ versus $-4.76 \mathrm{D}$ and -0.23 versus $-0.19 \mathrm{D}$, respectively). A more likely explanation of the differing results may be due to limited experience of surgeons in our study. The retrospective analysis and a small sample size were our primary limitations. Given that we had 61 eyes at our 3-month analysis and 41 eyes in our 6-month analysis, a larger sample size would have provided us with a greater statistical power. A recent study by Titiyal et al. [40] highlighted the significantly reduced surgical complications and speedier visual recovery 
for the surgeon after the initial 50 cases. The FDA study had nearly five times as many cases as our study (329 versus 68 , respectively). Thus, the larger number of cases in the FDA study compared to ours may have helped improve their visual outcomes by a small margin. Since our visual outcomes are consistent to other published SMILE results, it may be that the FDA study, with their larger number of cases, represents the significant potential improvement in visual outcomes simply from gaining more experience.

Dry eyes continues to be one of the most significant reported visual symptom postoperatively in refractive surgery [41]. Because SMILE is minimally invasive on the anterior corneal surface, it can be argued that SMILE should theoretically produce fewer dry eye symptoms than LASIK. Our analysis highlights that dry eyes were most significantly reported early in the postoperative interval and decreased in severity with time. Patient reported outcomes from the newest LASIK platforms in our previous study have illustrated a similar trend [42]. A meta-analysis by Denoyer et al. [43] comparing dry eye disease after SMILE versus LASIK, determined that SMILE had a lower incidence of dry eye disease, and consequently, an improved quality of life [43]. In their study, 23\% more patients in the LASIK group needed to use eye drops for their dry eyes than in the SMILE group at the 6-month postoperative interval [43]. Additionally, our analysis shows the prevalence of halos, glares, fluctuation, and depth perception were improved in a subset of our patient cohort. We could not find any other studies demonstrating an improvement of these symptoms after SMILE surgery. In fact, there is limited data overall regarding patient reported outcomes following SMILE. Given that the ultimate goal in any refractive surgery is to improve quality of life, we feel future direction should consistently include results of visual symptoms as part of the overall assessment on certain platforms and/or surgical techniques.

There are several limitations to our study. The retrospective analysis and a small sample size were our primary limitations. Given that we had 61 eyes at our 3-month analysis and 41 eyes in our 6-month analysis, a larger sample size would have provided us with a greater statistical power. The learning curve associated with SMILE along with the use of multiple surgeons may have slightly affected our overall outcomes. Furthermore, instead of using standardized questionnaires to report subjective visual symptoms, patients were asked about their symptoms during the physician-patient encounter. Our study was only able to collect this data from half our initial sample size. Thus, a limitation of this technique is that physicians may forget to ask certain questions or document certain findings. Additionally, there are two important biases in play with such an interview. One is the observer-expectancy bias in which a physician is less likely to document a certain visual symptom if he or she expects a positive outcome. The other is the Hawthorne effect, where patients may under report postoperative visual symptoms in an effort to please their physician. Having patients fill out an additional questionnaire anonymously through an independent agency, away from their physician, may offset some of these biases. We strongly recommend the use of validated questionnaires (i.e., Ocular Surface Disease Index and National Eye Institute's Refractive error Quality of Life [44-46]) in all postoperative refractive surgery follow-ups since a standardized comparison will aid surgeons in choosing the appropriate refractive surgery technique to optimize patient outcomes and will help guide future direction for improvement.

\section{Improvements}

Current studies utilizing even lower laser energy levels show that improved visual acuity and decreased aberrations are possible [47]. For instance, $\mathrm{Li}$ et al. [48] performed a recent retrospective study demonstrating that laser energy at $125 \mathrm{~nJ}$ with a spot-track-distance of $4.5 \mathrm{um}$ was the optimal combination in producing superior postoperative UDVA results in comparison to our results which were based on laser energy of 145 nJ. Furthermore, experimentation of SMILE with an infusion of a balanced salt solution may decrease coma and improve astigmatic correction [49]. Surgical 
experience may also play a role in faster visual recovery. With more time and experience, specific surgeon nomograms may help reduce the need for enhancements and potential undercorrection as well. When comparing our first 12 cases with our last 12 cases, spherical equivalence in the last 12 cases were closer to emmetropia, thus demonstrating that the procedure may improve outcomes with more surgeon experience $[16,50]$. As more patient data is analyzed, software will become more refined with improved adjustments for higher myopia since these patients tend to have undercorrection $[13,14,51]$.

\section{CONCLUSION}

SMILE has progressively become a more prevalent refractive surgery since its first clinical trials in 2011. This single-site experience shows that SMILE achieved good visual acuity and refractive outcomes. Overall, it performed favorably when compared to FDA PMA data and existing international SMILE outcomes. Our findings were superior to early generation excimer platforms for LASIK, however inferior to the latest excimer platforms, partially due to the inability to correct for low astigmatism. With upgraded software and laser technology, and increased surgeon experience with the procedure, SMILE outcomes will likely improve over time similar to past refractive procedures as demonstrated by our study.

\section{ACKNOWLEDGEMENTS}

Funding. This study was funded by an unrestricted Grant from Research to Prevent Blindness (RPB), 360 Lexington Avenue, 22nd Floor New York, NY 10017. No support was received for the publication of this article.

Authorship. All named authors meet the international Committee of Medical Journal Editors (ICMJE) criteria for authorship for this manuscript, take responsibility for the integrity of the work, and have given final approval to the version to be published.

Disclosures. Majid Moshirfar, Michael S. Murri, Tirth J. Shah, Steven H. Linn, Yasmyne Ronquillo, Orry C. Birdsong and Phillips C. Hoopes Jr have nothing to disclose.

Compliance with Ethical Guidelines. All procedures performed in studies involving human participants were in accordance with the ethical standards of the institutional and/or national research committee and with the 1964 Helsinki declaration and its later amendments or comparable ethical standards. Informed consent was obtained from all individual participants included in the study.

Open Access. This article is distributed under the terms of the Creative Commons Attribution-NonCommercial 4.0 International License (http://creativecommons.org/licenses/ by-nc/4.0/), which permits any noncommercial use, distribution, and reproduction in any medium, provided you give appropriate credit to the original author(s) and the source, provide a link to the Creative Commons license, and indicate if changes were made.

\section{REFERENCES}

1. Krueger RR, Juhasz T, Gualano A, Marchi V. The picosecond laser for nonmechanical laser in situ keratomileusis. J Refract Surg. 1998;14(4):467-9.

2. Ito M, Quantock AJ, Malhan S, Schanzlin DJ, Krueger RR. Picosecond laser in situ keratomileusis with a 1053-nm Nd:YLF laser. J Refract Surg. 1996;12(6):721-8.

3. Reinstein DZ, Archer TJ, Gobbe M. Small incision lenticule extraction (SMILE) history, fundamentals of a new refractive surgery technique and clinical outcomes. Eye Vis. 2014;1(1):3. https://doi.org/10. 1186/s40662-014-0003-1.

4. Moshirfar M, McCaughey MV, Reinstein DZ, Shah R, Santiago-Caban L, Fenzl CR. Small-incision lenticule extraction. J Cataract Refract Surg. 2015;41(3):652-65. https://doi.org/10.1016/j.jcrs. 2015.02.006. 
5. Shah R, Shah S, Sengupta S. Results of small incision lenticule extraction: all-in-one femtosecond laser refractive surgery. J Cataract Refract Surg. 2011;37(1):127-37. https://doi.org/10.1016/j.jcrs. 2010.07.033.

6. Yildirim Y, Olcucu O, Alagoz C, et al. Visual and refractive outcomes of photorefractive keratectomy and small incision lenticule extraction (SMILE) for myopia. J Refract Surg. 2016;32(9):604-10. https:// doi.org/10.3928/1081597X-20160602-02.

7. Reinstein DZ, Carp GI, Archer TJ, Gobbe M. Outcomes of small incision lenticule extraction (SMILE) in low myopia. J Refract Surg. 2014;30(12):812-8. https://doi.org/10.3928/ 1081597X-20141113-07.

8. Khalifa MA, Ghoneim A, Shafik Shaheen M, Aly MG, Piñero DP. Comparative analysis of the clinical outcomes of SMILE and wavefront-guided LASIK in low and moderate myopia. J Refract Surg. 2017;33(5):298-304. https://doi.org/10.3928/ 1081597X-20170222-01.

9. Zhu X, Zou L, Yu M, Qiu C, Chen M, Dai J. Comparison of postoperative visual quality after SMILE and LASEK for high myopia: a 1-year outcome. PLoS One. 2017. https://doi.org/10.1371/journal.pone. 0182251.

10. Burazovitch J, Naguzeswski D, Beuste T, Guillard M. Predictability of SMILE over four years in high myopes. J Fr Ophtalmol. 2017;40(6):e201-9. https://doi.org/10.1016/j.jfo.2017.05.001.

11. Liu YC, Rosman M, Mehta JS. Enhancement after small-incision lenticule extraction. incidence, risk factors, and outcomes. Ophthalmology. 2016;124(6):813-21. https://doi.org/10.1016/j. ophtha.2017.01.053.

12. Kim JR, Kim BK, Mun SJ, Chung YT, Kim HS. Oneyear outcomes of small-incision lenticule extraction (SMILE): mild to moderate myopia vs. high myopia. BMC Ophthalmol. 2015. https://doi.org/10.1186/ s12886-015-0051-x.

13. Wu W, Wang Y, Zhang H, Zhang J, Li H, Dou R. One-year visual outcome of small incision lenticule extraction (SMILE) surgery in high myopic eyes: retrospective cohort study. BMJ Open. 2016;6(9):e010993. https://doi.org/10.1136/ bmjopen-2015-010993.

14. Fernández J, Valero A, Martínez J, Piñero DP, Rodríguez-Vallejo M. Short-term outcomes of smallincision lenticule extraction (SMILE) for low, medium, and high myopia. Eur J Ophthalmol. 2016. https://doi.org/10.5301/ejo.5000849.
15. Ganesh S, Gupta R. Comparison of visual and refractive outcomes following femtosecond laserassisted LASIK with SMILE in patients with myopia or myopic astigmatism. J Refract Surg. 2014;30(9):590-6. https://doi.org/10.3928/ 1081597X-20140814-02.

16. Kim JR, Hwang HB, Mun SJ, Chung YT, Kim HS. Efficacy, predictability, and safety of small incision lenticule extraction: 6-months prospective cohort study. BMC Ophthalmol. 2014. https://doi.org/10. 1186/1471-2415-14-117.

17. Xu Y, Yang Y. Small-incision lenticule extraction for myopia: results of a 12-month prospective study. Optom Vis Sci. 2015;92(1):123-31. https:// doi.org/10.1097/OPX.0000000000000451.

18. Hansen RS, Lyhne N, Grauslund J, Vestergaard AH. Small-incision lenticule extraction (SMILE): outcomes of 722 eyes treated for myopia and myopic astigmatism. Graefe's Arch Clin Exp Ophthalmol. 2016;254(2):399-405. https://doi.org/10.1007/ s00417-015-3226-5.

19. Chansue E, Tanehsakdi M, Swasdibutra S, McAlinden C. Safety and efficacy of VisuMax ${ }^{\circledR}$ circle patterns for flap creation and enhancement following small incision lenticule extraction. Eye Vis. 2015;2(1):21. https://doi.org/10.1186/s40662-0150031-5.

20. Kamiya K, Shimizu K, Igarashi A, Kobashi H. Visual and refractive outcomes of femtosecond lenticule extraction and small-incision lenticule extraction for myopia. Am J Ophthalmol. 2014. https://doi. org/10.1016/j.ajo.2013.08.011.

21. Blum M, Täubig K, Gruhn C, Sekundo W, Kunert KS. Five-year results of small incision lenticule extraction (ReLEx SMILE). Cornea. 2016. https:// doi.org/10.1136/bjophthalmol-2015-306822.

22. Shen Z, Shi K, Yu Y, Yu X, Lin Y, Yao K. Small incision lenticule extraction (SMILE) versus femtosecond laser-assisted in situ keratomileusis (FSLASIK) for myopia: a systematic review and metaanalysis. PLoS One. 2016;11(7):e0158176. https:// doi.org/10.1371/journal.pone.0158176.

23. Holladay JT. Visual acuity measurements. J Cataract Refract Surg. 2004;30(2):287-90. https://doi.org/10. 1016/j.jcrs.2004.01.014.

24. Carl Zeiss Meditec Inc. Summary of safety and effectiveness data (SSED): Femtosecond Laser System for refractive correction; Visumax Femtosecond Laser.

25. United States Food and Drug Administration. Summary of Safety and Effectiveness Data (P930034/S13). https://www.accessdata.fda.gov/ 
cdrh_docs/pdf/P930034S013B.pdf. Accessed 15 May 2018.

26. United States Food and Drug Administration. Summary of Safety and Effectiveness Data (P970053/S002). https://www.accessdata.fda.gov/ cdrh_docs/pdf/P970053S002B.pdf. Accessed 15 May 2018.

27. United States Food and Drug Administration. Summary of Safety and Effectiveness Data (P990010). https://www.accessdata.fda.gov/cdrh_ docs/pdf/P990010B.pdf. Accessed 15 May 2018.

28. United States Food and Drug Administration. Summary of Safety and Effectiveness Data (P930016/S045). https://www.accessdata.fda.gov/ cdrh_docs/pdf/P930016S045B.pdf. Accessed 15 May 2018.

29. United States Food and Drug Administration. Summary of Safety and Effectiveness Data (P970053/S011). https://www.accessdata.fda.gov/ cdrh_docs/pdf/P970053S011B.pdf. Accessed 15 May 2018.

30. United States Food and Drug Administration. Summary of safety and effectiveness data (P020050/ S12). https://www.accessdata.fda.gov/cdrh_docs/ pdf2/P020050S012B.pdf. Accessed 15 May 2018.

31. Peyman G. Ophthalmology calculator. http://www. drpeyman.ir/Ophthalmology_Calculator.htm. Retreived 10 May 2018.

32. Waring GO, Reinstein DZ, Dupps WJ Jr, Kohnen T, Mamalis N, Rosen ES, Koch DD, Obstbaum SA, Stulting RD. Standardized graphs and terms for refractive surgery results. J Refract Surg. 2011;27(1):7-9.

33. Sandoval HP, Donnenfeld ED, Kohnen T, et al. Modern laser in situ keratomileusis outcomes. J Cataract Refract Surg. 2016;42:1224-34. https:// doi.org/10.1016/j.jcrs.2016.07.012.

34. Sekundo W, Kunert KS, Blum M. Small incision corneal refractive surgery using the small incision lenticule extraction (SMILE) procedure for the correction of myopia and myopic astigmatism: results of a 6 month prospective study. Br J Ophthalmol. 2011;95(3):335-9. https://doi.org/10.1136/bjo. 2009.174284.

35. Dong Z, Zhou X. Irregular astigmatism after femtosecond laser refractive lenticule extraction. J Cataract Refract Surg. 2013;39(6):952-4. https:// doi.org/10.1016/j.jcrs.2013.04.016.

36. Ganesh S, Brar S, Arra RR. Refractive lenticule extraction small incision lenticule extraction: a new refractive surgery paradigm. Indian J Ophthalmol.
2018;66(1):10-9. https://doi.org/10.4103/ijo.IJO_ 761_17.

37. Alió del Barrio JL, Vargas V, Al-Shymali O, Alió JL. Small incision lenticule extraction (SMILE) in the correction of myopic astigmatism: outcomes and limitations-an update. Eye Vis. 2017;4(1):26. https://doi.org/10.1186/s40662-017-0091-9.

38. Chan TCY, Ng ALK, Cheng GPM, et al. Vector analysis of astigmatic correction after small-incision lenticule extraction and femtosecond-assisted LASIK for low to moderate myopic astigmatism. Br J Ophthalmol. 2016;100(4):553-9. https://doi.org/ 10.1136/bjophthalmol-2015-307238.

39. Zhang J, Wang Y, Chen X. Comparison of moderate-to high-astigmatism corrections using wavefront-guided laser in situ keratomileusis and smallincision lenticule extraction. Cornea. 2016;35(4): 523-30. https://doi.org/10.1097/ICO.00000000000 00782 .

40. Titiyal JS, Kaur M, Rathi A, Falera R, Chaniyara M, Sharma N. Learning curve of small incision lenticule extraction. Cornea. 2017;36(11):1377-82. https://doi.org/10.1097/ICO.0000000000001323.

41. Toda I. LASIK and the Ocular Surface. Cornea. 2008. https://doi.org/10.1097/ico.0b013e31817f42c0.

42. Moshirfar M, Shah TJ, Skanchy DF, Linn SH, Durrie DS. Meta-analysis of the FDA reports on patientreported outcomes using the three latest platforms for LASIK. J Refract Surg. 2017;33(6):362-8. https:// doi.org/10.3928/1081597X-20161221-02.

43. Denoyer A, Landman E, Trinh L, Faure JF, Auclin F, Baudouin C. Dry eye disease after refractive surgery: comparative outcomes of small incision lenticule extraction versus LASIK. Ophthalmology. 2015;122(4):669-76. https://doi.org/10.1016/j.opht ha.2014.10.004.

44. Schiffman RM. Reliability and validity of the ocular surface disease index. Arch Ophthalmol. 2000;118(5):615. https://doi.org/10.1001/archopht. 118.5.615.

45. Mangione CM, Lee PP, Pitts J, Gutierrez P, Berry S, Hays RD. Psychometric properties of the National Eye Institute visual function questionnaire (NEIVFQ). Arch Ophthalmol. 1998;116(11):1496-504.

46. McDonnell PJ, Mangione C, Lee P, et al. Responsiveness of the national eye institute refractive error quality of life instrument to surgical correction of refractive error. Ophthalmology. 2003;110(12): 2302-9. https://doi.org/10.1016/j.ophtha.2003.02. 004. 
47. Ji YW, Kim M, Kang DSY, et al. Lower laser energy levels lead to better visual recovery after small-incision lenticule extraction: prospective randomized clinical trial. Am J Ophthalmol. 2017;179:159-70. https://doi.org/10.1016/j.ajo.2017.05.005.

48. Li L, Schallhorn JM, Ma J, Cui T, Wang Y. Energy Setting and visual outcomes in SMILE: a retrospective cohort study. J Refract Surg. 2018;34(1):11-6. https://doi.org/10.3928/1081597X-20171115-01.

49. Liu T, Zhu X, Chen K, Bai J. Visual outcomes after balanced salt solution infiltration during lenticule separation in small-incision lenticule extraction for myopic astigmatism. Med. 2017;96(30):e7409.

50. Taneri S, Kießler S, Rost A, Dick B. Experience with introduction of SMILE: learning phase of our first 200 treatments. Klin Monbl Augenheilkd. 2016. https://doi.org/10.1055/s-0042-114040.

51. Jin HY, Wan T, Wu F, Yao K. Comparison of visual results and higher-order aberrations after small incision lenticule extraction (SMILE): High myopia vs. mild to moderate myopia. BMC Ophthalmol. 2017. https://doi.org/10.1186/s12886-017-0507-2. 